Systèmes dynamiques/Dynamical systems

Équations différentielles/Ordinary Differential Equations

\title{
La non-intégrabilité méromorphe du problème plan des trois corps
}

\section{Tsygvintsev Alexei}

Laboratoire de Mathématiques Emile Picard, C.N.R.S.- UMR 5580, Université Paul Sabatier, 118, route de Narbonne, 31062 Toulouse Cédex 4, France

Courriel : tsygvin@picard.ups-tlse.fr

et Section de Mathématiques, Université de Genève, 2-4, rue du Lièvre, Case postale 240, CH-1211 Genève 24, Suisse

Courriel : Alexei.Tsygvintsev@math.unige.ch

Résumé. Nous considérons le problème des trois corps dans le plan et montrons que ce problème ne possède pas de système complet d'intégrales premières méromorphes à un voisinage de la solution particulière de Lagrange.

The meromorphic non-integrability of the planar three-body problem

Abstract.We study the planar three-body problem and prove the absence of a complete set of complex meromorphic first integrals in a neighborhood of the Lagrangian solution.

\section{Introduction et résultats}

Considérons trois corps $P_{1}, P_{2}, P_{3}$ sur le plan avec des masses $m_{1}, m_{2}, m_{3}$ qui s'attirent conformément à la loi de Newton. Soient $\left(x_{1}, x_{2}\right)$ les coordonnées de $P_{1},\left(x_{3}, x_{4}\right)$ les coordonnées de $P_{2},\left(x_{5}, x_{6}\right)$ les coordonnées de $P_{3}$. Notons $y_{r}=m_{k} \frac{d x_{r}}{d t}$ où $k$ est la partie entière de $(r+1) / 2$.

Les équations de Hamilton du mouvement des corps prennent la forme

$$
\frac{d x_{r}}{d t}=\frac{\partial H_{1}}{\partial y_{r}}, \quad \frac{d y_{r}}{d t}=-\frac{\partial H_{1}}{\partial x_{r}}, \quad(r=1,2, \ldots, 6),
$$

avec l'Hamiltonien

$$
\begin{aligned}
& H_{1}=\frac{1}{2 m_{1}}\left(y_{1}^{2}+y_{2}^{2}\right)+\frac{1}{2 m_{2}}\left(y_{3}^{2}+y_{4}^{2}\right)+\frac{1}{2 m_{3}}\left(y_{5}^{2}+y_{6}^{2}\right)-m_{3} m_{2}\left\{\left(x_{3}-x_{5}\right)^{2}+\left(x_{4}-x_{6}\right)^{2}\right\}^{-1 / 2} \\
& -m_{3} m_{1}\left\{\left(x_{5}-x_{1}\right)^{2}+\left(x_{6}-x_{2}\right)^{2}\right\}^{-1 / 2}-m_{1} m_{2}\left\{\left(x_{1}-x_{3}\right)^{2}+\left(x_{2}-x_{4}\right)^{2}\right\}^{-1 / 2} .
\end{aligned}
$$

Ce problème peut être illustré par l'attraction mutuelle de la terre, de la lune et du soleil.

Les intégrales premières connues du système (1.1) sont $F_{1}=H_{1}-$ l'énergie,

$F_{2}=y_{1}+y_{3}+y_{5}, F_{3}=y_{2}+y_{4}+y_{6}$ - les intégrales du mouvement du centre de gravité, $F_{4}=y_{1} x_{2}+y_{3} x_{4}+y_{5} x_{6}-x_{1} y_{2}-x_{3} y_{4}-x_{5} y_{6}=k-$ l'intégrale des aires.

En supposant que le centre de gravité est fixe, c'est-à-dire que $F_{2}=F_{3}=0$, et en utilisant l'intégrale des aires $F_{4}$, le système (1.1) peut être ramené à 3 degrés de liberté [6]

$$
\frac{d q_{r}}{d t}=\frac{\partial H}{\partial p_{r}}, \quad \frac{d p_{r}}{d t}=-\frac{\partial H}{\partial q_{r}}, \quad(r=1,2,3),
$$

où

$$
\begin{aligned}
& H=\frac{M_{1}}{2}\left\{p_{1}^{2}+\frac{1}{q_{1}^{2}} P^{2}\right\}+\frac{M_{2}}{2}\left(p_{2}^{2}+p_{3}^{2}\right)+\frac{1}{m_{3}}\left\{p_{1} p_{2}-\frac{p_{3}}{q_{1}} P\right\}-\frac{m_{1} m_{3}}{r_{1}}-\frac{m_{3} m_{2}}{r_{2}}-\frac{m_{1} m_{2}}{r_{3}} \\
& P=p_{3} q_{2}-p_{2} q_{3}-k
\end{aligned}
$$


et

$$
r_{1}=q_{1}, \quad r_{2}=\sqrt{q_{2}^{2}+q_{3}^{2}}, \quad r_{3}=\sqrt{\left(q_{1}-q_{2}\right)^{2}+q_{3}^{2}},
$$

sont les distances mutuelles des corps.

Nous appellerons le système (1.2) le problème plan des trois corps.

D'après Bruns [1] le problème (1.1) et donc le problème (1.2) n'admet pas d'intégrale première algébrique, autre que les intégrales premières déja connues.

Poincaré [4] a démontré en 1892 que si la troisième masse $m_{3}=\mu$ est infiniment petite et est attireé par les deux premières masses $m_{1}$ et $m_{2}$, alors le problème (1.2) n'admet pas d'intégrale première holomorphe en $\mu$ autre que celle de l'énergie.

Il est bien connu (Lagrange [2]) que ce problème possède une solution particulière dans laquelle les trois corps forment un triangle équilatéral et décrivent chacun une conique. Notons $\Gamma$ celle qui correspond au cas parabolique.

Nous considérons dans cette Note la question de l'existence de deux intégrales premières supplementaires méromorphes et fonctionnellement indépendantes autres que celle de l'énergie au voisinage de la solution $\Gamma$.

Notre résultat principal est le suivant:

THÉORÈME 1.1- Le problème plan des trois corps n'admet pas deux intégrales premières supplementaires méromorphes par rapport aux variables $q_{i}, p_{j}, r_{k}$ et fonctionnellement indépendantes au voisinage de la solution particulière de Lagrange $\Gamma$.

COROLLAIRE 1.2- Le problème plan des trois corps n'est pas complètement méromorphiquement intégrable au voisinage de la solution $\Gamma$.

La preuve est basée sur la théorie de Ziglin [7] qui donne une condition nécessaire de nonintégrabilité.

Dans le cas de masses égales, le même résultat a été obtenu par D. Boucher à l'aide du théorème de non-intégrabilité de Moralis-Ramis [3].

\section{Les équations normales variationnelles le long de la solution $\Gamma$}

Dans le cas parabolique la solution de Lagrange peut être écrite sous la forme suivante

$$
\left(q_{1}, q_{2}, q_{3}\right)=\left(q, \frac{q}{2}, \frac{\sqrt{3} q}{2}\right), \quad\left(p_{1}, p_{2}, p_{3}\right)=\left(p, \alpha p+\frac{\beta}{q}, \gamma p+\frac{\delta}{q}\right)
$$

où $\alpha, \beta, \gamma, \delta$ sont les constantes.

Pour $q(t), p(t)$ on a

$$
q=P(w), \quad p=\frac{w}{P(w)}, \quad P(w)=e_{1} w^{2}+e_{2} w+e_{3}
$$

avec les constantes $e_{1}, e_{2}, e_{3}$.

Nous avons donc la paramétrisation de la solution de Lagrange

$$
\Gamma=\left\{q_{i}(w), p_{i}(w) \mid w \in \mathbf{C P}^{\mathbf{1}}\right\}
$$

A partir de cette solution particulière, on peut calculer les équations normales variationnelles (voir [5]) qui prennent la forme d'un système fuchsien 


$$
\frac{d x}{d \tau}=\left(\frac{A}{\tau-\tau_{0}}+\frac{B}{\tau-\tau_{1}}+\frac{C}{\tau-\tau_{2}}\right) x, \quad x \in \mathbf{C}^{4},
$$

où $\tau_{0}, \tau_{1}, \tau_{2} \in \mathbf{C}$ sont les singularités et $A, B, C$ sont les $4 \times 4$ matrices constantes dépendantes sur des masses $m_{1}, m_{2}, m_{3}$.

\section{Le groupe de monodromie des équations (2.1)}

Soit $G$ le groupe de monodromie des équations (2.1).

Nous désignons $T_{i}, T_{\infty}, i=0,1,2$ les générateurs de $G$ correspondants respectivement aux groupes de monodromie locaux autour les singularités $\tau_{i}, i=0,1,2$ et $\tau=\infty$.

LEMME 3.1

a) $T_{0}=I$ - est l'élément neutre de $G$.

b) Les générateurs $T_{1}, T_{2}$ ont la même forme de Jordan

$$
\left(\begin{array}{llll}
1 & 1 & 0 & 0 \\
0 & 1 & 0 & 0 \\
0 & 0 & 1 & 1 \\
0 & 0 & 0 & 1
\end{array}\right) .
$$

c) Le générateur $T_{\infty}$ a les valeurs propres suivantes

$$
\operatorname{Spectre}\left(T_{\infty}\right)=\left\{e^{2 \pi i \lambda_{1}}, \quad e^{2 \pi i \lambda_{2}}, \quad e^{-2 \pi i \lambda_{1}}, \quad e^{-2 \pi i \lambda_{2}}\right\}
$$

où

$$
\lambda_{1}=\frac{3}{2}+\frac{1}{2} \sqrt{13+\sqrt{\theta}}, \quad \lambda_{2}=\frac{3}{2}+\frac{1}{2} \sqrt{13-\sqrt{\theta}},
$$

et

$$
\theta=144\left(1-3 \frac{S_{2}}{S_{1}^{2}}\right), \quad S_{1}=m_{1}+m_{2}+m_{3}, \quad S_{2}=m_{1} m_{2}+m_{3} m_{2}+m_{1} m_{3} .
$$

COROLLAIRE 3.2

$$
T_{1} T_{2}=T_{\infty}^{-1}
$$

COROLLAIRE 3.3- On aura toujours

$$
\operatorname{Spectre}\left(T_{\infty}\right) \neq\{1,1,1,1\}
$$

Esquisse de démonstration.- Pour tout les singularités $\tau_{i}, \tau=\infty, i=0,1,2$ on peut calculer des solution formelles locales du système (2.1) et trouver donc les générateurs de $G$ dans chaque point singulier.

\section{Esquisse de la démonstration du théorème 1.1}

Notre démonstration est inspirée par Ziglin [7]. Supposons que le problème plan des trois corps (1.2) possède deux intégrales premières supplementaires méromorphes par rapport aux variables $q_{i}$, $p_{j}, r_{k}$ et fonctionnellement indépendantes au voisinage de la solution particulière de Lagrange $\Gamma$. Nous en déduisons le lemme suivant 
LEMME 4.1 (Ziglin [7])-Le groupe de monodromie $G$ des équations normales variationnelles (2.1) a deux invariants rationnels et fonctionnellement indépendantes $J_{1}(x), J_{2}(x)$.

En vertu du lemme 3.1 on peut supposer sans perte de généralité que

$$
T_{1}=\left(\begin{array}{cccc}
1 & 1 & 0 & 0 \\
0 & 1 & 0 & 0 \\
0 & 0 & 1 & 1 \\
0 & 0 & 0 & 1
\end{array}\right)=I+D
$$

où

$$
D=\left(\begin{array}{llll}
0 & 1 & 0 & 0 \\
0 & 0 & 0 & 0 \\
0 & 0 & 0 & 1 \\
0 & 0 & 0 & 0
\end{array}\right)
$$

On aura pour la matrice $T_{2}$

$$
T_{2}=I+R
$$

où

$$
R=V^{-1} D V=\left(\begin{array}{cccc}
a_{1} & a_{2} & a_{3} & a_{4} \\
b_{1} & b_{2} & b_{3} & b_{4} \\
c_{1} & c_{2} & c_{3} & c_{4} \\
d_{1} & d_{2} & d_{3} & d_{4}
\end{array}\right)
$$

avec une matrice $V, \operatorname{det}(V) \neq 0$ et les constantes inconnues $a_{i}, b_{i}, c_{i}, d_{i} \in \mathbf{C}$.

Remarque 4.2. La matrice étant nilpotente,

$$
\operatorname{Spectre}(R)=\{0,0,0,0\} .
$$

LEMME 4.3- Soit $J$ l'invariant rationnel de $G$, alors

$$
\delta J=0, \quad \Delta J=0,
$$

où $\delta=x_{2} \frac{\partial}{\partial x_{1}}+x_{4} \frac{\partial}{\partial x_{3}}, \quad \Delta=\left(\sum_{i=1}^{4} a_{i} x_{i}\right) \frac{\partial}{\partial x_{1}}+\left(\sum_{i=1}^{4} b_{i} x_{i}\right) \frac{\partial}{\partial x_{2}}+\left(\sum_{i=1}^{4} c_{i} x_{i}\right) \frac{\partial}{\partial x_{3}}+\left(\sum_{i=1}^{4} d_{i} x_{i}\right) \frac{\partial}{\partial x_{4}}$.

Démonstration du lemme 4.3.- Pour un arbitraire $n \in \mathbf{N}$ nous avons $T_{1}^{n}=I+n D$. Par conséquent

$$
J\left(T_{1}^{n} x\right)=J(x+n D x)=J(x)+n \delta J(x)+\sum_{i=2}^{\infty} n^{i} r_{i}(x)=J(x),
$$

Il résulte de là que $\delta J=0$. La preuve de l'identité $\Delta J=0$ s'effectue de la même manière.

On obtient donc

$$
\delta J_{i}=\Delta J_{i}=0, \quad i=1,2 .
$$

Ces équations nous permettent de trouver les restrictions sur les paramètres $a_{i}, b_{i}, c_{i}, d_{i}$ qui s'écrivent sous la forme d'un systéme d'équations algébriques

$$
\begin{gathered}
P_{1}(a, b, c, d)=0, \\
\cdot \cdot \cdot \\
P_{m}(a, b, c, d)=0,
\end{gathered}
$$


avec les polynômes $P_{1} \ldots P_{m}, m \in \mathbf{N}$.

Par ailleurs, la condition 4.1 nous donne un autre système d'équations sur les mêmes paramètres

$$
\begin{gathered}
L_{1}(a, b, c, d)=0, \\
\cdot \cdot \cdot \\
L_{n}(a, b, c, d)=0,
\end{gathered}
$$

avec les polynômes $L_{1} \ldots L_{n}, n \in \mathbf{N}$.

On peut vérifier par un calcul direct, que pour $a_{i}, b_{i}, c_{i}, d_{i}$ satisfaisant aux systèmes (4.2) et (4.3) on aura toujours

$$
\operatorname{Spectr}\left(T_{1} T_{2}\right)=\{1,1,1,1\},
$$

d'où, en utilisant le corollaire 3.2

$$
\operatorname{Spectr}\left(T_{\infty}\right)=\{1,1,1,1\}
$$

Or cela est contraire au corollaire 3.3. Ceci achève la démonstration du théorème 1.1.

\section{Remerciements}

Je remercie L. Gavrilov and V. Kozlov pour m'avoir proposé le sujet de ce travail et ses suggestions. Je veux également remercier J.-P. Ramis, J.-A. Weil et D. Bucher pour l'intérêt qu'ils ont porté à ce travail.

\section{Références bibliographiques}

[1] H. Bruns, Ueberdie Integrale des vierkörper Problems, Acta Math. 11, p. 25-96, 1887.

[2] J.L. Lagrange, Oeuvres. Vol. 6, 272-292, Paris, 1873.

[3] J.J. Morales-Ruiz, J.P. Ramis, Galosian Obstructions to integrability of Hamiltonian Systems, Preprint, 1998.

[4] H. Poincaré, Les méthodes novelles de la mécanique céleste, vol. 1, chap. 5, Paris,1892.

[5] A. Tsygvintsev, On the variational equations of three-body problem near Lagrangian solutions, Prépublication 150 du Laboratoire de Mathématiques E. Picard, Université Toulouse III, 1999.

[6] E.T. Whittaker, A Treatise on the Analytical Dynamics of particles and Rigid Bodies, Cambridge University Press, New York, 1970.

[7] S.L. Ziglin, Branching of solutions and non-existence of first integrals in Hamiltonian Mechanics I, Func. Anal. Appl. 16, 1982. 